\title{
EXTRAGALACTIC PLANETARY NEBULAE
}

\author{
Chairperson and Editor: Y. TERZIAN
}




\title{
EXTRAGALACTIC PLANETARY NEBULAE
}

\author{
SIDNEY VAN DEN BERGH \\ Dominion Astrophysical Observatory \\ National Research Council \\ 5071 West Saanich Road \\ Victoria, B.C., V8X 4M6 \\ Canada
}

\section{Introduction}

It is a great pleasure for me to be able to start off this "Joint Discussion" on Extragalactic Planetary Nebulae. Many astronomers appear somewhat ambivalent in their attitudes towards planetary nebulae. A few of us regard planetaries as the spectacular "last hurrah" before stars slide off into oblivion as white dwarfs. Others think of them as soaring butterflies that evolved from stellar caterpillars. Finally a few less romantic astronomers simply ask "what can planetary nebulae do for us?" Perhaps surprisingly the answer is that they can do a great deal for the progress of various branches of astronomy. At this meeting George Jacoby will, for example, show us how planetary nebulae can be used to measure extragalactic distances. Mike Dopita, Ziaohui Hui and Ken Freeman will tell us that planetaries are powerful probes of the kinematics of galaxies. Furthermore Henry Ferguson will show how planetary nebulae can be used to probe the effects of metallicity on stellar evolution in elliptical galaxies.

\section{Luminosities of planetary nebulae}

Fig. 1 shows a comparison of the distances of individual planetary nebulae derived by Cahn, Kaler \& Stanghellini (1992) and by Zhang \& Kwok (1993). In view of the very poor agreement found for distances to individual Galactic planetaries it is ironic (Aller 1993) that Fig. 2 (Jacoby et al. 1992) shows such good agreement between distances of galaxies derived form the luminosity functions of planetary nebulae and those obtained from surface brightness fluctuations. 


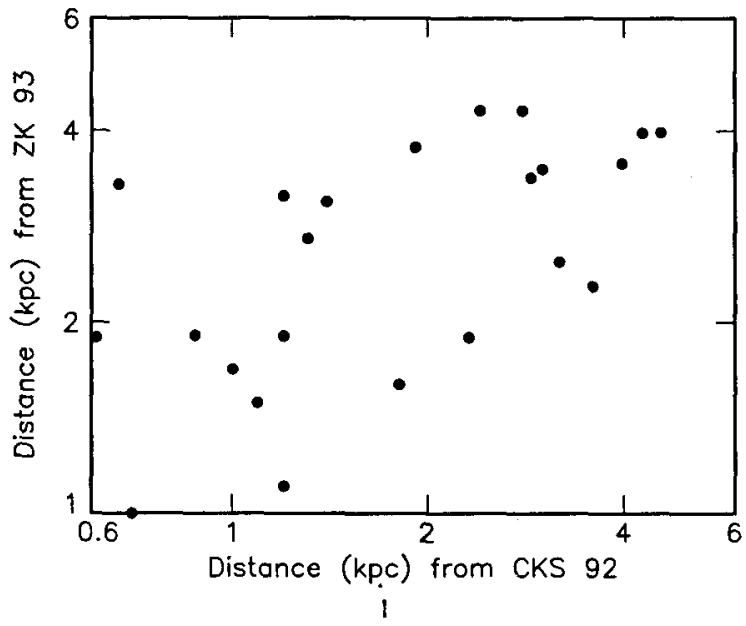

Fig. 1 Intercomparison of distances to individual planetary nebulae obtained by Cahn, Kaler \& Stanghellini (1992) and by Zhang \& Kwok (1993)

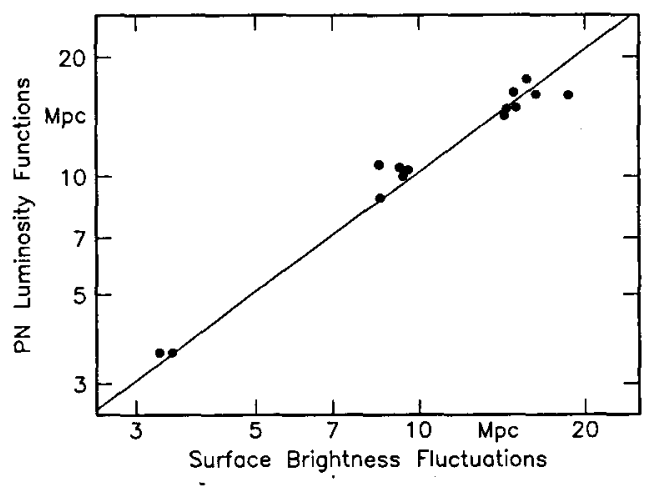

Fig. 2 Intercomparison of distances to galaxies derived from the luminosity functions of planetary nebulae and from galactic surface brightness fluctuations. Figure adapted from Jacoby et al. (1992).

\section{Birthrate of planetary nebulae}

Fig. 3 shows a plot of the birthrate of planetary nebulae versus $M_{b o l}$, from data compiled by Peimbert (1990). The observations of luminous (mainly early-type) galaxies may be represented by the relation

$$
\dot{\xi} \approx 5.1 \times 10^{-12}\left(\mathrm{M}_{\mathrm{bol}}-23.7\right),
$$

in which $\mathrm{M}_{\text {bol }}$ is the bolometric magnitude of the parent galaxy and $\xi$ is the formation rate of planetary nebulae expressed in $\mathrm{yr}^{-1} \mathrm{~L}_{\circ}^{-1}$. Eqn.(1) indicates that the rate of planetary nebula formation decreases rapidly with increasing galaxy luminosity and vanishes for galaxies brighter 


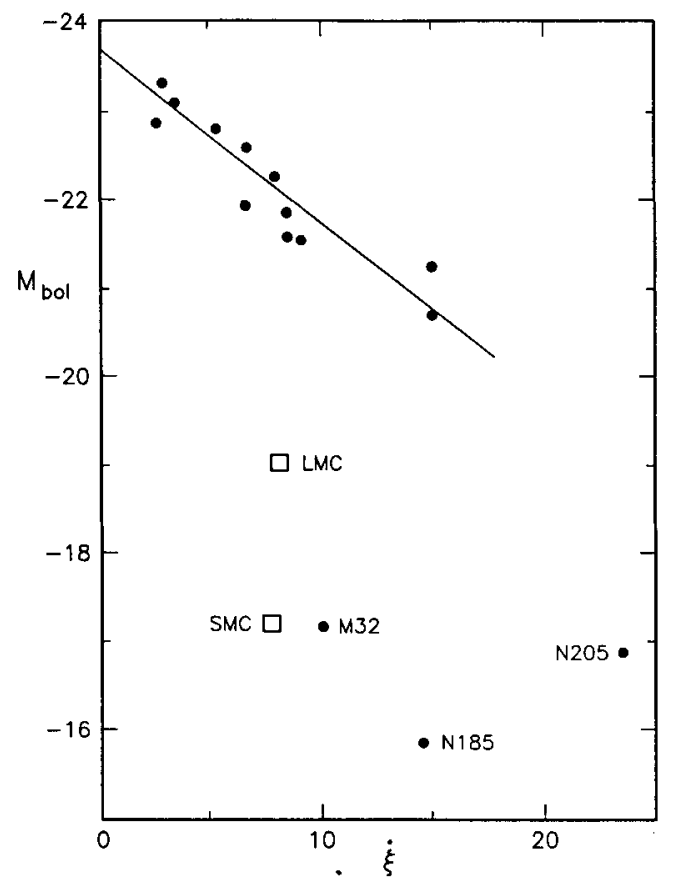

Fig. 3 Dependence of the rate of formation of planetary nebulae on parent galaxy luminosity. Note that the specific formation rate of planetaries decreases with increasing galaxy luminosity, and hence presumably, with increasing metallicity.

than $\mathbf{M}_{\mathrm{bol}} \approx-23.7$. The observation that the most luminous early-type galaxies have the strongest UV upturns (Greggio \& Renzini 1990), and hence presumably the largest number of extreme horizontal branch stars, suggests (Clegg 1993, Dorman, O'Connell \& Rood 1994) that mass loss, which is a function of metallicity, may determine if evolved stars become extreme horizontal branch stars (AGB stars manque) or normal asymptotic branch stars that evolve into planetary nebulae.

\section{Galaxy kinematics}

Since planetary nebulae exhibit emission spectra (with rather narrow lines) their radial velocities can be determined easily and accurately. Planetaries are therefore almost ideal probes for study of the kinematics and dynamics of early-type galaxies. The potential of this technique has recently been demonstrated by papers on NGC 3379, NGC 4472, NGC 4486 and NGC 5128 (see Ciardullo, Jacoby \& Dejonghe 1993 for references). Additional studies of the kinematics of early-type galaxies based on planetary nebulae, which will be reported at this meeting, are eagerly awaited.

Finally observations of planetary nebulae can be used to investigate abundance gradients, and hence the evolutionary histories, of galaxies. Using this technique Pasquali \& Perinotto (1993) have, for example, found that our own galaxy was possibly more inhomogeneous in the past than it is at the present time. Similarly detailed investigations of planetaries in other galaxies would clearly be very exciting. 


\section{References}

Aller, L.H. 1993 in Planetary Nebulae = IAU Symposium No. 155, eds. R. Weinberger and A. Acker (Kluwer Dordrect), 1

Cahn, J.H. Kaler, J.B., \& Stanghellini, L. 1992, A\&AS, 94, 399

Ciardullo, R., Jacoby, G.H., \& Dejonghe, H.B. 1993, ApJ, 414, 454

Clegg, R.E.S. 1993 in Planetary Nebulae = IAU Symposium No. 155, eds. R. Weinberger and A. Acker (Kluwer, Dordrecht), 549

Dorman, B., O'Connell, R.W., \& Rood, R.T. 1994, in preparation

Greggio, L., \& Renzini, A. 1990, ApJ, 364, 35

Jacoby, G.H., Branch, D., Ciardullo, R., Davies, R.L., Harris, W.E., Pierce, M.J., Pritchet, C.J., Tonry, J.L., \& Welch, D.L. 1992, PASP, 104, 599

Pasquali, A., \& Perinotto, M. 1993, A\&A, 280, 581

Peimbert, M. 1990 Rev. Mex. Astroph. 20, 119

Zhang, C.Y., \& Kwok, S. 1993 ApJS, 88, 137 